\title{
Favre-Averaged Non-Linear Harmonic Method for Compressible Periodic Flows
}

\author{
Feng Wang* and Luca di Mare ${ }^{\dagger}$ \\ Oxford Thermo-fluids Institute, University of Oxford, Oxford, United Kingdom, OX2 OES
}

The treatment of compressible flows subject to periodic disturbances of finite amplitude with non-linear harmonic (NLH) methods requires a careful choice of averaging procedure and the variable set to average - A good choice of averaging procedure and its associated variable is one that preserves certain properties of the flow of particular interest and also leads to a simple formulation of the equations governing the time-mean flow. This paper studies the time-averaged Navier-Stokes equations with different averaging procedure and variable set, and presents a new formulation of the non-linear harmonic method based on Favre-averaging of the primitive variable set. The resulting formulation of the time-averaged Navier-Stokes equations have exactly the same form as the steady base flow plus contributions from deterministic fluxes and stresses. Favre averages of the primitive variables preserve the time average of the conserved flow variables. From a practical point of view, this can minimize the modifications to the baseline solver to implement the non-linear harmonic method. The details of the method are presented and the method is validated from a quasi-2D transonic diffuser to compressors in subsonic and transonic flow regimes. Good agreement with the unsteady simulations using time-marching are observed for all cases.

\section{Nomenclature}

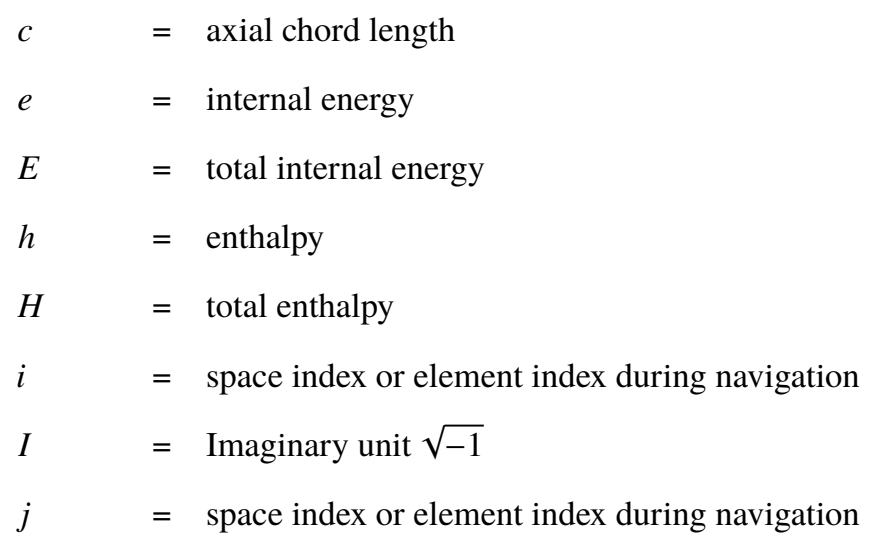

\footnotetext{
* Research associate, Oxford Thermofluids Institute

'Associate professor, Oxford Thermofluids Institute
} 


$$
\begin{aligned}
& l=\text { harmonic index during navigation } \\
& M R T P=\text { corrected mass flow } \\
& n=\text { iteration index of pseudo time marching } \\
& N=\text { number of harmonics } \\
& p \quad=\text { pressure } \\
& P_{r} \quad=\text { total-to-total pressure ratio } \\
& q \quad=\text { primitive flow variable } \\
& R \quad=\text { gas constant } \\
& S \quad=\quad \text { strain rate } \\
& t \quad=\text { time } \\
& T=\text { temperature } \\
& u \quad=\text { velocity } \\
& \mathbf{U}=\text { conservative variable } \\
& x=\text { coordinate } \\
& \delta_{i j} \quad=\quad \text { Kronecker delta } \\
& \varepsilon=\text { magnitude of flow perturbation } \\
& \rho \quad=\text { density } \\
& \omega=\text { angular frequency } \\
& \Omega \quad=\text { Volume } \\
& \kappa=\text { heat conduction coefficient } \\
& \tau_{i j} \quad=\text { laminar shear stress tensor } \\
& \tau_{i j}^{t} \quad=\quad \text { Reynolds shear stress tensor } \\
& \phi \quad=\text { dummy variable }
\end{aligned}
$$

Subscripts and superscripts

$$
\begin{aligned}
& \quad=\text { deterministic fluctuation to the time-averaged mean flow } \\
& \quad=\text { deterministic fluctuation to the density-averaged mean flow } \\
& * \quad=\text { pseudo time } \\
& \text { Operator } \\
& -\quad=\text { time-averaging } \\
& \sim \quad=\text { density averaging }
\end{aligned}
$$




\section{Introduction}

Computational fluid dynamics (CFD) is widely used in the design of fluid machinery. Most design calculations consist of steady-state simulations based on the Favre/Reynolds averaged Navier-stokes (NS) equations. However, most flows encountered in fluid machinery (e.g. gas turbine) are inherently unsteady and it is actually the unsteadiness of the flow that allows a machine to exchange energy with the working fluid.

For the purpose of representing unsteady flows, it is convenient to decompose each flow variable into a mean value, a stochastic fluctuation and a periodic (or deterministic) fluctuation. The stochastic fluctuations are due to turbulence and their effect on the mean flow is represented by additional momentum and energy fluxes usually referred to as Reynolds stress and heat flux. If the Boussinesq approximation is used, the effect of the Reynolds stress and heat flux are evaluated based on a local eddy viscosity and the mean flow velocity and temperature gradients. Reynolds stress and its work done on the fluid represent the enhanced momentum and energy transfer due to turbulence.

The deterministic fluctuation is caused by periodic variations in flow conditions, such as the wakes of upstream bladerows in a turbo-machine. For the periodic fluctuation, a second averaging over one period can be applied and this will yield terms which have similar form to the ones due to the stochastic fluctuations. For incompressible flows, the energy equation is decoupled from the mass and momentum equations, and the deterministic stresses are the main effect of the periodic fluctuation on the mean flow. For compressible flows, there are also extra terms in the energy equations.

In the following text, assuming the effect of the stochastic fluctuation on the mean flow is already approximated by a turbulence model, the phrase "unsteady effect" refers to the effect of the deterministic fluctuation on the mean flow. The extra terms due to the deterministic fluctuation are termed as deterministic fluxes in this paper, since they appear as fluxes from a finite volume point of view.

Adamczyk modeled the deterministic fluxes by assuming the deterministic flow field was transported by an axisymmetric background flow [1] and his method was successfully applied to aerodynamic design [2]. The method, however, was non-trivial to implement with a general mesh [3]. An alternative approach was devised by $\mathrm{He}$ and Ning [4]. The general idea was that instead of modeling the deterministic fluxes they could be computed directly via computationally efficient unsteady computations. Since the fluctuations were periodic, it was convenient to represent them through a truncated Fourier series. The unsteady problem was then cast into the frequency domain as a set of steady linearised problems. For a multistage simulation, only one passage was required for each blade row and a phase shift was applied on the periodic boundaries. Deterministic fluxes were then evaluated from the Fourier coefficients and inserted into the governing equations of the mean flow. The mean flow and the linearized NS solver were coupled together, therefore this approach was termed as the Non-linear Harmonic (NLH) method. Later on, the coupling of the harmonics were included [5] and the clocking effect was also considered [6].

Similar to He's method, Hall et al. [7] proposed the harmonic balance method. As the name of the method indicated, the cross-coupling of harmonics was considered. Following Hall's approach, a few variants of the harmonic balance 
method were proposed, such as the time-spectral method by Sicot et al. [8] and the nonlinear frequency domain method by McMullen et al. [9]. Mathematically these variants of the harmonic balance method were similar and it was only a matter of preference of storing the flow variables as Fourier coefficient or time level form [10]. The harmonic balance method, however, does not preserve flux continuity exactly when used for multistage simulations with a finite number of harmonics. Moreover, since the cross-coupling of the harmonics is considered, special treatment of aliasing is required.

Minimum modifications to the baseline solver can be achieved to implement the harmonic balance method, such as the two-step approximate factorization approach by Thomas et al [11]. However, more effort is required to implement the the NLH method. One needs a dedicated linearized NS solver to compute the flow perturbations, the deterministic fluxes needs to be included in the baseline solver. Furthremore, He and Ning's [4] method was originally formulated for the conserved variables, and an explicit conversion is needed whenever primitive variables are needed.

One favorable feature of the NLH is that it can provide a hierarchy of modeling fidelity. Since it is not always necessary to consider the cross coupling of the harmonics in the simulation [10], one could decouple the linearized flow from the mean flow when the unsteady flow is essentially linear, and also consider the cross-coupling of the harmonics for strong non-linear flows (e.g. strong shocks) [12]. Furthermore flux conservation can be enforced at the blade row interface [13], independently of the harmonics in the linearized solver. This is a favorable feature because at the design stage in order to save computational cost one probably only wants to run a handful of harmonics to capture the effect of the deterministic perturbations on the mean flow while flux conservations should be satisfied at the blade row interface.

There is only limited literature on NLH, such as the work of Vilmin et al [12, 14, 15]. The present paper addresses the problem of a suitable relation between the choice of variables used to represent the flow field and the precise meaning of the time averaging adopted in NLH and similar methods. The present paper argues that this relation is set by the very nature of the conservation equations and that if judicious choices are made, considerable simplification can be achieved over the procedure shown by He and Ning [4].

This paper is organized as the following: Section $\Pi$ discusses the fundamental issue of averaging unsteady flows, the formulations of the time-averaged flow regarding to different combination of averaging procedure and variable set, and the most suitable combinations for NLH method, Section III details the methodology of the Favre-averaged non-linear harmonic method, Section $\mathrm{IV}$ shows the validation of the method on a transonic diffuser, quasi-3D stator-rotor configuration and 3D multistage compressor.

\section{Averaging Periodic Disturbance in Compressible Flow}

In this section, we discuss the procedure of averaging periodic compressible flows and its impact on the resulting time-averaged system, and in particular on the relation between the base flow for the purpose of linearization and the time average of the unsteady solution.

Let $\mathbf{q}_{\mathbf{0}}$ denote a known flow field and $\mathbf{q}^{\prime}=\varepsilon \hat{\mathbf{q}} e^{I \omega t}$ a harmonic perturbation superimposed on $\mathbf{q}_{\mathbf{0}}$. In the linear limit 
$\varepsilon \rightarrow 0, \mathbf{q}_{\mathbf{0}}$ is not only the time mean of the unsteady flow but also the state around which the the equations of motion can be linearised to study the evolution of the perturbation field $\mathbf{q}^{\prime}$. Furthermore, in the linear limit any function $f(\mathbf{q})$ has average $\overline{f(\mathbf{q})}=f(\overline{\mathbf{q}})$. As an example, for the conserved variables $\overline{\mathbf{U}}=\mathbf{U}(\overline{\mathbf{q}})$. Additionally, in the linear limit, all statements of the conservation principles are equivalent, apart from consideratons relating to the discretization.

For finite amplitude fluctuations the identity between time mean and reference state for linearization is lost in general. As an example, the conserved variables $\mathbf{U}$ no longer obey $\overline{\mathbf{U}}=\mathbf{U}(\overline{\mathbf{q}})$. Furthermore, the time averages of the conservation equations are not identically satisfied by any set of time averaged solutions. Take the one dimensional Euler equation as an example:

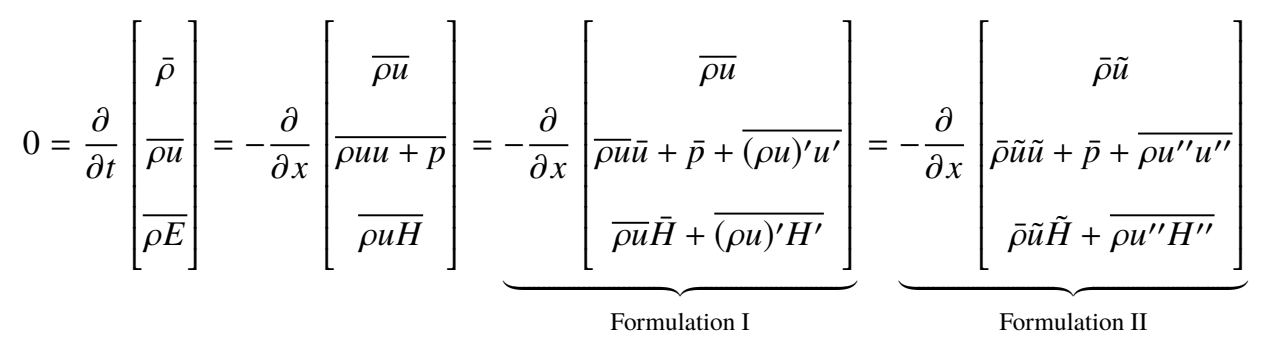

The bar represents time-averages and the tilde Favre-averages (or density-averages), which are defined as $\tilde{\phi}=\frac{\overline{\rho \phi}}{\bar{\rho}}$. The superscript ' represents the fluctuation with respect to the time-averaged variable and the one " represents the fluctuation with respect to the density-averaged variable. It is clear, therefore, that for compressible flows under periodic disturbances of finite amplitude, the choice of averaging procedure and the variable set leads to different time-averaged system.

If the conservative variables are chosen, time-averaging arises naturally from the time-averaged Euler equations in conservative form, as is shown in Formulation I of Equation 1 . However, even the time averaged conservation equations contain terms depending the time-averaged primitive variables, which requires both the time-averaged conservative variables and the fluctuations to work out. For example,the time-averaged velocity, pressure, enthalpy and the square of the speed of sound can be computed as:

$$
\begin{aligned}
\bar{u}_{i} & =\frac{1}{\bar{\rho}}\left(\overline{\rho u_{i}}-\overline{\rho^{\prime} u_{i}^{\prime}}\right) \\
\bar{p} & =(\gamma-1)\left(\overline{\rho E}-\frac{1}{2}\left(\overline{\rho u} \bar{u}+\overline{(\rho u)^{\prime} u^{\prime}}\right)\right. \\
\bar{H} & =\frac{1}{\bar{\rho}}\left(\overline{\rho E}-\overline{\rho^{\prime} E^{\prime}}\right)+\bar{p} \frac{\overline{1}}{\rho}+\overline{p^{\prime}\left(\frac{1}{\rho}\right)^{\prime}} \\
\overline{a^{2}} & =\gamma\left(\bar{p} \frac{\overline{1}}{\rho}+\overline{p^{\prime}\left(\frac{1}{\rho}\right)^{\prime}}\right)
\end{aligned}
$$

It can been that the above time-averaged quantities are in a more complicated form compared to the fashion they are computed in a steady flow. This complexity is due to the non-linearity of the NS equations and the averaging procedure. 
An alternative averaging procedure can lead to simpler forms.

Favre-averages [16, 17] of the primitive variables arise naturally from the time averages of the conservation equations if the fluxes are expressed as functions of the primitive variables themselves. The unsteady flow can represented as:

$$
\begin{aligned}
& u_{i}=\tilde{u}_{i}+u_{i}^{\prime \prime} \\
& \rho=\bar{\rho}+\rho^{\prime} \\
& p=\bar{p}+p^{\prime} \\
& h=\tilde{h}+h^{\prime \prime} \\
& e=\tilde{e}+e^{\prime \prime} \\
& T=\tilde{T}+T^{\prime \prime}
\end{aligned}
$$

It is noted that density and pressure are time-averaged and the rest are Favre-averaged. Assume the air is perfect gas, the time-averaged equation of state can be written as $\bar{p}=\bar{\rho} R \tilde{T}$ and the Favre-averaged internal energy and enthalpy can be computed as $\tilde{e}=\frac{1}{\gamma-1} \frac{\bar{p}}{\bar{\rho}}$ and $\tilde{h}=\frac{\gamma}{\gamma-1} \frac{\bar{p}}{\bar{\rho}}$ respectively. It is also worth mentioning that $\overline{u^{\prime \prime}} \neq 0$ but $\overline{\rho u^{\prime \prime}}=0$. For a detailed description of Favre-averaging, readers can refer to Wilcox [16] or Cebeci [17]. The Favre-averaged velocity, pressure, enthalpy and speed of sound can be computed as:

$$
\begin{aligned}
\tilde{u}_{i} & =\frac{\overline{\rho u}}{\bar{\rho}} \\
\bar{p} & =(\gamma-1) \overline{\rho e} \\
\tilde{H} & =\tilde{E}-\frac{\bar{p}}{\bar{\rho}} \\
\tilde{a^{2}} & =\gamma \frac{\bar{p}}{\bar{\rho}}
\end{aligned}
$$

It is noted that the square of speed of sound are density-averaged and the Favre-averaged speed of sound is defined as $\tilde{a}=\sqrt{\tilde{a}^{2}}=\gamma \overline{\bar{p}}$. These formulations are considerably simpler compared to Equation 2 which are based on time-averaging conservative variables. Moreover they are formally identical to the corresponding relations for steady flow.

Therefore it is clear that it is advantageous to use Favre-averages and primitive variables to formulate the timeaveraged equations. Such a procedure will be a more suitable procedure for the non-linear harmonic method compared to the original procedure of $\mathrm{He}$ and Ning [4]. The resulting time-averaged system will have exactly the same formulation as the steady flow plus the contribution of the deterministic fluxes. For a practitioner who is interested in implementing NLH, compared to the original method Favre-averages will lead to minimum modifications to the baseline solver. The details of this reformulation is detailed in the next section. 


\section{Favre-averaged Non-linear Harmonic Method}

\section{A. Basic Formulation}

The starting point is the unsteady RANS equations for compressible flows, the differential form of which is shown in Equation 7 . The effect of the stochastic perturbations on the mean flow has been approximated by the turbulence models and the interaction between the stochastic perturbation and the periodic perturbation is not considered.

$$
\begin{gathered}
\frac{\partial \rho}{\partial t}+\frac{\partial\left(\rho u_{i}\right)}{\partial x_{j}}=0 \\
\frac{\partial\left(\rho u_{i}\right)}{\partial t}+\frac{\partial\left(\rho u_{i} u_{j}\right)}{\partial x_{j}}=-\frac{\partial p}{\partial x_{i}}+\frac{\partial}{\partial x_{j}}\left(\tau_{i j}+\tau_{i j}^{t}\right) \\
\frac{\partial(\rho E)}{\partial t}+\frac{\partial\left(\rho u_{j} H\right)}{\partial x_{j}}=-\frac{\partial}{\partial x_{j}}\left(\kappa \frac{\partial T}{\partial x_{j}}+u_{i}\left(\tau_{i j}+\tau_{i j}^{t}\right)\right)
\end{gathered}
$$

where $\tau_{i j}^{t}$ is the Reynolds stress tensor. Turbulent kinetic energy has been included in the total energy $E$ and total enthalpy $H$.

Substituting the flow decompositions of Equation 4 into Equation 7 followed by Favre-averaging, this results in the system for the mean flow. Due to the non-linearity of the NS equations, there are extra terms which appear in the momentum and energy equations due to the periodic perturbations. These terms represent the effects of the periodic perturbations on the mean flow. The resulting system is shown in Equation 8 .

$$
\begin{gathered}
\frac{\partial \bar{\rho}}{\partial t^{*}}+\frac{\partial\left(\bar{\rho} \tilde{u}_{i}\right)}{\partial x_{j}}=0 \\
\frac{\partial\left(\bar{\rho} \tilde{u}_{i}\right)}{\partial t^{*}}+\frac{\partial\left(\bar{\rho} \tilde{u}_{i} \tilde{u}_{j}\right)}{\partial x_{j}}=-\frac{\partial \bar{p}}{\partial x_{i}}+\frac{\partial}{\partial x_{j}}\left(\tilde{\tau}_{i j}+\tilde{\tau}_{i j}^{t}-\overline{\rho u_{i}^{\prime \prime} u_{j}^{\prime \prime}}\right) \\
\frac{\partial}{\partial t^{*}}(\bar{\rho} \tilde{E})+\frac{\partial}{\partial x_{j}}\left(\bar{\rho} \tilde{u}_{j} \tilde{H}+\overline{\rho u_{i}^{\prime \prime} H^{\prime \prime}}\right)=-\frac{\partial}{\partial x_{j}}\left(\kappa \frac{\partial \tilde{T}}{\partial x_{j}}\right)+\frac{\partial}{\partial x_{j}}\left[\tilde{u}_{i}\left(\tilde{\tau}_{i j}+\tau_{i j}^{t}\right)\right]
\end{gathered}
$$

Where $t^{*}$ is the pseudo time for convergence purpose only and it has no physical meaning, $\tilde{\tau}_{i j}=2 \mu\left(\tilde{S}_{i j}-\frac{1}{3} \frac{\partial \tilde{u}_{k}}{\partial x_{k}} \delta_{i j}\right)$ and $\bar{p}=\bar{\rho} R \tilde{T}$.

The extra terms are due to the non-linearity of the NS equations after the averaging. The deterministic kinetic energy $k_{D}=\frac{1}{2} \overline{u_{i}^{\prime \prime} u_{i}^{\prime \prime}}$ is already included in $\tilde{E}$ and $\tilde{H}$. Other extra terms appear as fluxes. These extra fluxes are termed as deterministic fluxes. The extra term in the momentum equation $\overline{\rho u_{i}^{\prime \prime} u_{j}^{\prime \prime}}$ has similar form to the Reynolds stresses, so it is called deterministic stresses in the literature [1]. It represents the momentum transfer due to the periodic fluctuations. In the energy equation the term $\overline{\rho u_{i}^{\prime \prime} H^{\prime \prime}}$ represents the re-distribution of total enthalpy due to periodic fluctuations.

The system for the periodic flow perturbations can be obtained by the difference of Equation 7 and Equation 8 . 
By discarding the high order terms, a system similar to the original NLH is obtained and this is shown in Equation 9 Despite the similarity, there is a fundamental difference. The flow is now linearized around the Favre-averaged flow and the flow perturbations are defined in Equation 4, while the original NLH is linearized around the time-averaged conservative variables.

$$
\begin{aligned}
\frac{\partial \bar{\rho}}{\partial t^{*}}+\frac{\partial\left(\rho u_{i}\right)^{\prime \prime}}{\partial x_{j}} & =0 \\
\frac{\partial}{\partial t^{*}}\left(\rho u_{i}\right)^{\prime \prime}+\frac{\partial}{\partial x_{j}}\left(\left(\rho u_{i}\right)^{\prime \prime} \tilde{u}_{j}+\bar{\rho} \tilde{u}_{i} u_{j}^{\prime \prime}\right) & =-\frac{\partial p^{\prime \prime}}{\partial x_{i}}+\frac{\partial}{\partial x_{j}}\left(\tau_{i j}^{\prime \prime}+\tau_{i j}^{t \prime \prime}\right) \\
\frac{\partial}{\partial t^{*}}(\rho E)^{\prime \prime}+\frac{\partial}{\partial x_{j}}\left(\bar{\rho} \tilde{u}_{i} H^{\prime \prime}+(\rho H)^{\prime \prime} \tilde{u}_{i}\right) & =-\frac{\partial}{\partial x_{j}}\left(\kappa \frac{\partial T^{\prime \prime}}{\partial x_{j}}\right)+\frac{\partial}{\partial x_{j}}\left[\tilde{u}_{i}\left(\tau_{i j}^{\prime \prime}+\tau_{i j}^{t \prime \prime}\right)+u_{i}^{\prime \prime}\left(\tilde{\tau}_{i j}+\tilde{\tau}_{i j}^{t}\right)\right]
\end{aligned}
$$

Where $t^{*}$ is the pseudo time for convergence purpose only and it has no physical meaning. Perturbations of conservative variables in Equation 9 can be defined as:

$$
(\rho \phi)^{\prime \prime}=\bar{\rho} \phi^{\prime \prime}+\tilde{\phi} \rho^{\prime \prime}
$$

The periodic perturbations of the primitive variables can be approximated by truncated Fourier series as:

$$
\phi^{\prime \prime}=\sum_{l=-N}^{l=N} \hat{\phi}_{l} e^{-I \omega_{l} t}
$$

Where $l$ is the harmonic index, $N$ is the number of harmonics, $I$ is the imaginary unit, $\omega_{l}$ is the angular frequency of the $l^{\text {th }}$ harmonic and $t$ is time.

Substituting Equation 11 into Equation 9 will lead to a set of coupled $N$ equations for the Fourier coefficients of $\hat{\phi}_{l}$. If only the first order terms are kept, which means the cross-coupling of the harmonics are ignored, the harmonics will be decoupled from each other and the equation for each harmonic is independent. In this work, the cross-coupling of the harmonics is not included. As will be presented in the next section, the effect of the cross-coupling is found to be negligible for many practical applications. The exception is when the flow is strongly non-linear (e.g. strong shock), the effect of the cross-coupling should be considered.

When the periodic flow perturbation is available, the deterministic fluxes can be evaluated directly. For example, the deterministic stress $\overline{\rho u_{i}^{\prime \prime} u_{j}^{\prime \prime}}$ can be written as $\bar{\rho} \overline{u_{i}^{\prime \prime} u_{j}^{\prime \prime}}$ by ignoring the triple term $\overline{\rho^{\prime} u_{i}^{\prime \prime} u_{j}^{\prime \prime}} . \overline{u_{i}^{\prime \prime} u_{j}^{\prime \prime}}$ can be computed as:

$$
\overline{u_{i}^{\prime \prime} u_{j}^{\prime \prime}}=2 \sum_{l=1}^{l=N}\left[\operatorname{Re}\left(\hat{u}_{i}^{l}\right) \operatorname{Re}\left(\hat{u}_{j}^{l}\right)+\operatorname{Im}\left(\hat{u}_{i}^{l}\right) \operatorname{Im}\left(\hat{u}_{j}^{l}\right)\right]
$$

The current approach differs from the original NLH on the definitions of averaging and the resulting coupled system. 
In order to differentiate the current method from the original NLH method, the current method is called Favre-averaged NLH (FNLH) method.

\section{B. Solution Method}

The baseline solver is the in-house CFD solver AU3X [18, 19]. AU3X is written in C++ and solves the unsteady Reynolds-averaged Navier-Stokes equations using a fully implicit cell-centered finite volume scheme on unstructured or structured meshes. The flow gradients are evaluated with the weighted least-squares method. Inviscid fluxes are computed by Roe's approximate Riemann solver. Second-order accuracy in space discretization is achieved by the monotonic upstream-centered scheme for conservation laws (MUSCL) approach with the van Albada limiter. Steady and unsteady solutions are obtained via pseudo time-marching and dual-time stepping, respectively. The code finds its applications from intake-fan interactions [20, 21] to massive parallel whole engine gas path CFD simulations [19]. The code has recently been optimized on modern many-core architectures [22].

AU3X is solved with Newton-Jacobi iteration and exact linearization of the NS equations is already available. This functionality is reused to solve the complex magnitudes of the harmonics. In particular, the base solver uses Roe's Riemann solver to evaluate the convective fluxes and an exact linearization of Roe's Riemann solver is implemented. To achieve second-order spatial accuracy for the linearized NS, the MUSCL scheme is directly applied to the complex magnitudes of the harmonics. The gradient of the complex magnitudes are evaluated via the weighted least square method [23]. The complex magnitudes are then interpolated from the cell center to the cell interface using the Van Albada limiter.

The coupled system of the mean flow and the linearized NS for the $l^{\text {th }}$ harmonic is shown in Equation 13

$$
\begin{gathered}
\left(\frac{\Omega_{i}}{\Delta t} \delta_{i j}+\left.\sum_{j=1}^{j=N_{e}} \frac{\partial R_{i j}(\mathbf{U})}{\partial \mathbf{U}_{\mathbf{i}}}\right|_{\left.\mathbf{U}_{\mathbf{i}}^{\mathbf{n}}\right) \Delta \mathbf{U}_{\mathbf{i}}^{\mathbf{n}}}=\sum_{j=1}^{j=N_{e}}-R_{i j}\left(\mathbf{U}^{\mathbf{n}}\right)\right. \\
\left(\frac{\Omega_{i}}{\Delta t} \delta_{i j}+\left.\sum_{j=1}^{j=N_{e}} \frac{\partial R_{i j}(\mathbf{U})}{\partial \mathbf{U}_{\mathbf{i}}}\right|_{\mathbf{U}_{i}^{n}}+\left|\omega_{l}\right| \Omega_{i} \delta_{i j}\right) \Delta \hat{\mathbf{U}}_{\mathbf{i}}^{\mathbf{n}}=\sum_{j=1}^{j=N_{e}}\left(-\left.\frac{\partial R_{i j}(\mathbf{U})}{\partial \mathbf{U}_{\mathbf{i}}}\right|_{\mathbf{U}_{i}^{n}} \hat{\mathbf{U}}_{i}^{n}\right)-I \omega_{l} \Omega_{i} \hat{\mathbf{U}}_{i}^{n}
\end{gathered}
$$

Where $\Delta \mathbf{U}^{n}=\mathbf{U}^{n+1}-\mathbf{U}^{n+1}$ and $\Delta \hat{\mathbf{U}}^{n}=\hat{\mathbf{U}}^{n+1}-\hat{\mathbf{U}}^{n+1}$

$\left.\sum_{j=1}^{j=N_{e}} \frac{\partial R_{i j}(\mathbf{U})}{\partial \mathbf{U}_{i}}\right|_{\mathbf{U}_{i j}^{n}}$ is the flux Jacobian of cell $i$ accumulated on the interfaces with the neighboring cells. $N_{e}$ is the number of neighboring cells of cell $i$. The flux Jacobian appears on the left hand side (LHS) of the systems of the mean and linearized flows. In the current work, the flux Jacobian on the LHS is never evaluated explicitly but approximated by its spectra radius. The mean flow is solved implicitly with Newton-Jacobi. The flux $R_{i j}\left(\mathbf{U}^{n}\right)$ is linearized exactly for the mass, momentum and energy equations but approximated for the one-equation or two-equation turbulence models. The linearized fluxes in the mean flow are re-used by the linearized flows to assemble the right hand side (RHS) of the 
linearized NS solver. For the system of the linearized flow, the term $\left|\omega_{i}\right| \Omega_{i}$ is added to the diagonal part of the LHS to increase the spectra radius and the stability of numerical method. On the RHS, the term $-I \omega_{l} \Omega_{i} \hat{\mathbf{U}}_{i}^{n}$ is the spectral source term and treated explicitly.

The mean flow and the linearized flow are solved simultaneously via pseudo marching. To march the solution from time step $n$ to $n+1$, a few Newton iterations are used to march the solution of the mean flow. Meanwhile, for the linearized flow the approximated Jacobian from the mean flow is used, a 5-stage Runge-Kutta approach is used with the following coefficients [24] $\alpha_{i}=[0.0695,0.1602,0.2898,0.5060,1]$. This procedure is found to be numerically robust from simple 2D transonic diffusers to 3D multistage transonic and subsonic compressors.

\section{Boundary conditions}

\section{Inlet/exit boundary}

For simulations with multiple components, such as multistage turbomachinery simulations, each bladerow is meshed separately and the coupling among bladerows occurs through the interface. The approach is similar to the original approach by Chen et al. [13] but the formulation is recast in the framework of Favre-averaging. For the linear NS equation, the boundary condition at the inlet is obtained from the spatial FFT of the exit boundary of the upstream bladerow, and the boundary condition at the exit is from the spatial FFT of the inlet boundary of the downstream bladerow. Quasi-3D non-reflective boundary condition (NRBC) is applied to the inlet and the exit boundary for the mean flow and the linearized flow [25].

It is noted that non-reflective treatment is mandatory to ensure that the boundary condition for the linear NS of the neighboring bladerow is set up correctly. When 1D non-reflective treatment is used, the shape of the wake is distorted due to the reflection. Since the FFT of this wake profile will be used to set up the inlet boundary condition for the linear NS of the downstream bladerow, the phase and magnitude of the harmonics will not be trustworthy. In this work, the quasi-3D non-reflective treatment by Saxer and Giles [26] is used.

For the mean flow, assume the faces are already grouped into a set of bands and the faces of each band are on the same radial level. For simplicity assume the bands on both sides of the interface match with each other. If not, a conservative interpolation can be used. The mass, momentum and energy fluxes on either side of the bladerow interface on a band is evaluated as:

$$
\begin{array}{r}
\int_{\theta_{1}}^{\theta_{2}}\left(\bar{\rho} \tilde{u}_{n}\right) d S=F_{1} \\
\int_{\theta_{1}}^{\theta_{2}}\left(\bar{\rho} \tilde{u}_{n} \tilde{u}_{i}+\overline{\rho u_{n}^{\prime \prime} u_{i}^{\prime \prime}}+\bar{p} n_{i}\right) d S=F_{2, i} \\
\int_{\theta_{1}}^{\theta_{2}}\left(\bar{\rho} \tilde{u}_{n} \tilde{H}+\overline{\rho u_{n}^{\prime \prime} H^{\prime \prime}}\right) d S=F_{3}
\end{array}
$$


$F_{1}, F_{2, i}$ and $F_{3}$ will then be turned into flux averaged variables [27]. These variables are transfered to the neighboring bladerow. It is noted that mass is always conserved in this approach regardless of the number of harmonics to solve in the linearized NS.

\section{Periodic boundary}

For turbomachinery applications, only one passage is required for each bladerow. The periodic boundary condition for the mean is trivial. For the linearized NS, given an inter-blade phase angle (IBPA) $\sigma$ the phase-shift periodic boundary condition is applied. Assuming $\hat{q}_{L}$ is the flow variable on the lower periodic boundary, the flow variable on the upper periodic boundary can be worked out as:

$$
\hat{q}_{U}=\hat{q}_{L} e^{I \sigma}
$$

\section{Solid wall}

Non-slip conditions are used for both the mean flow and the linearized NS. For the mean flow, automatic wall treatment is used to make results insensitive with respect to wall mesh refinement [28]. At each iteration, $y^{+}$of the first cell on the wall is first computed. The wall shear stress is then computed in the region of law of wall $\left(y^{+}>11\right)$ or the laminar viscous sublayer $\left(y^{+} \leq 11\right)$. For the linearized NS, the $y^{+}$of the mean flow is used and the linearized wall shear stress is computed from the linearized formulas for the wall shear stress in the log law region or the viscous sublayer regions.

\section{Results}

\section{A. Sabjen Transonic Diffuser}

The first test case is a transonic diffuser experimentally studied by Sajben et al [29] and studied numerically by He and Ning [4]. The ratio of the back static pressure to the inlet total pressure is 0.82 and this produces a weak shock with a preshock Mach number of 1.24 at $x / H=1.4 . x$ is the axial distance from the inlet and $H$ is the height of the throat. The flow is assumed to be fully turbulent and the Wilcox $k-\omega$ turbulence model is used. The mesh and the contour of the Mach number are shown in Fig. 1. It can be seen that the boundary layer is separated due to the shock near the throat.

The predicted static pressure on the top and bottom walls for the steady simulation is compared with the experimental data. This is shown in Fig. 2. Two set of meshes are used. The first mesh has $120 \times 45$ elements and the the second mesh has $240 \times 45$ elements by doubling the number of cells in the streamwise direction. It can be seen that both meshes have credible predictions of the static pressure on both the top and bottom walls. The position of the shock is predicted satisfactorily. The differences of the predicted pressure on the top and bottom walls are marginal for both meshes. In the following study, the mesh with $120 \times 45$ elements is used. 


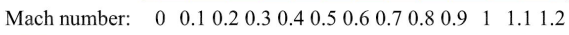

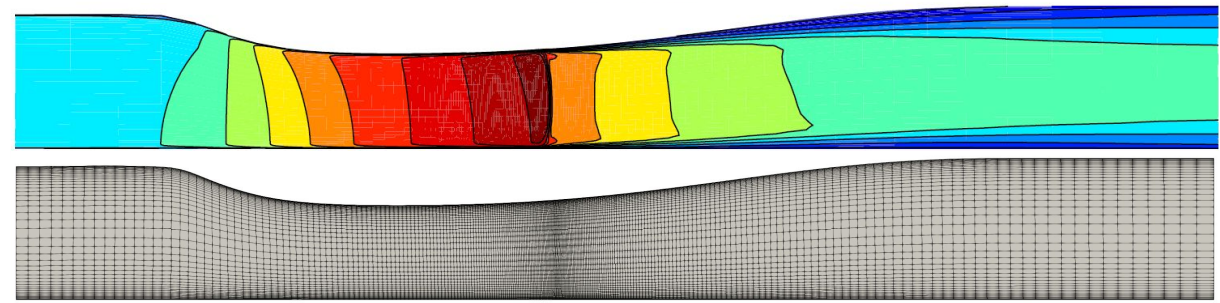

Fig. 1 Mach number contours and mesh details of the Sajben diffuser.
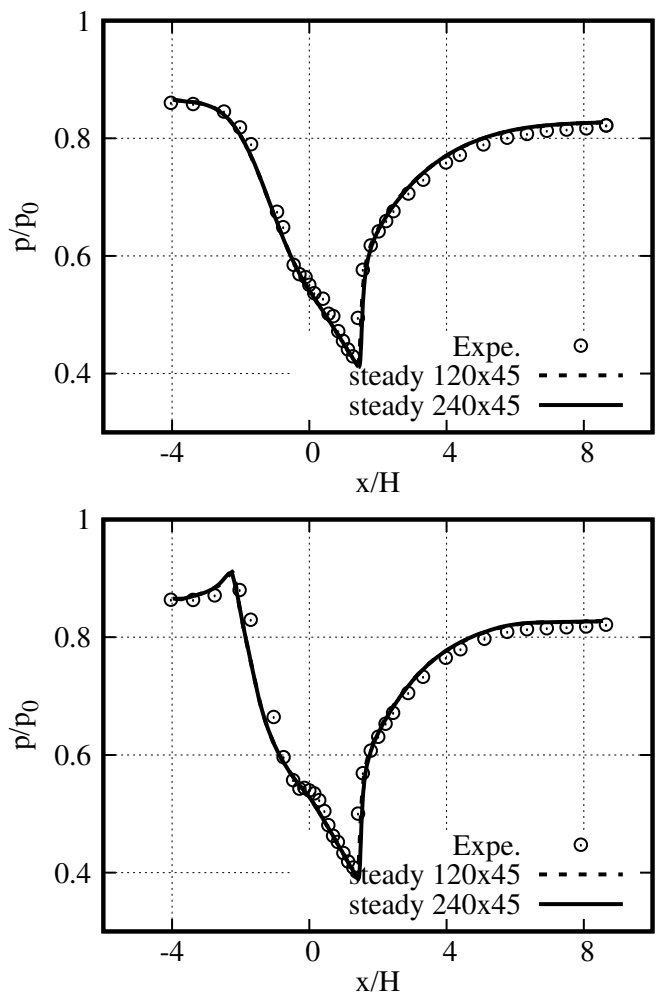

Fig. 2 Predicted pressure distribution on top and bottom walls. 


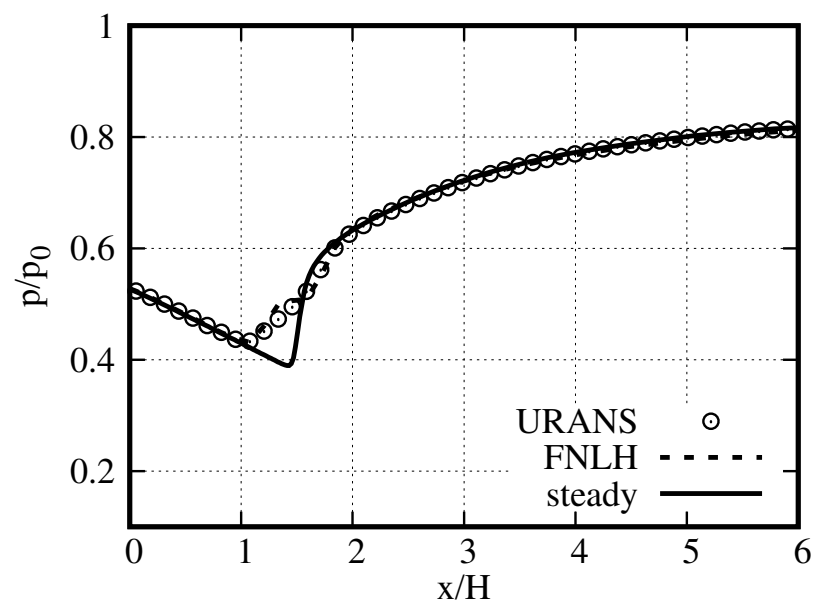

Fig. 3 Predicted pressure distribution on top wall of the diffuser by FNLH.

The back pressure of the diffuser is then oscillated harmonically as $p=p_{0}(1+\varepsilon \cos (2 \pi f t)) . f$ is $150 \mathrm{HZ}$ and $\varepsilon$ is $2 \%$. The URANS and the solution with the current method is compared and this is shown in Fig. 3 . Due to the non-linearity of the NS equation, the shock in the time-averaged solution at the throat is smeared and this is captured well with the current method. The discrepancy between the current method and the URANS is due to the cross-coupling of the harmonics. Previous researches [12] have reported that the agreement can be improved by including the cross-coupling of the harmonics.

\section{B. Quasi-3D subsonic Rotor-Stator Simulation}

The second test case is the middle span sections of a stator and rotor which are in the rear stages of a compressor studied by the authors previously [30]. Multi-block structured meshes are used for the simulations and the meshing technique can be found in Wang and di Mare [31]. The flows are in the subsonic flow regime and the airfoils are re-designed so that the stream surfaces where the airfoil is designed sit on constant radius. The blade count ratio is scaled to 2:1 to reduce the computational cost of the URANS simulations. The mesh for this study is shown in Fig 4 Since the non-reflective treatment for the URANS is not implemented in the current solver, the inlet boundary of the stator and the exit boundary of the rotor have been extended and the meshes around both regions are also coarsened to damp reflective waves.

Three sets of meshes are used for the rotor. From coarse to fine, the rotor has 16922, 25264 and 38720 elements respectively. The $y+$ of the coarse mesh is around 30 and the $y+$ for the medium and fine meshes are around 1 . The fine mesh has more points on the blade and more elements in the freestream, and the expansion ratio of the grid spacing close to the wall is decreased from 1.3 to 1.1 compared to the medium mesh. The computed unsteady pressure from these three meshes is shown in Fig. 5 . The difference between the medium and the fine mesh is marginal. In this study the medium mesh is used. 


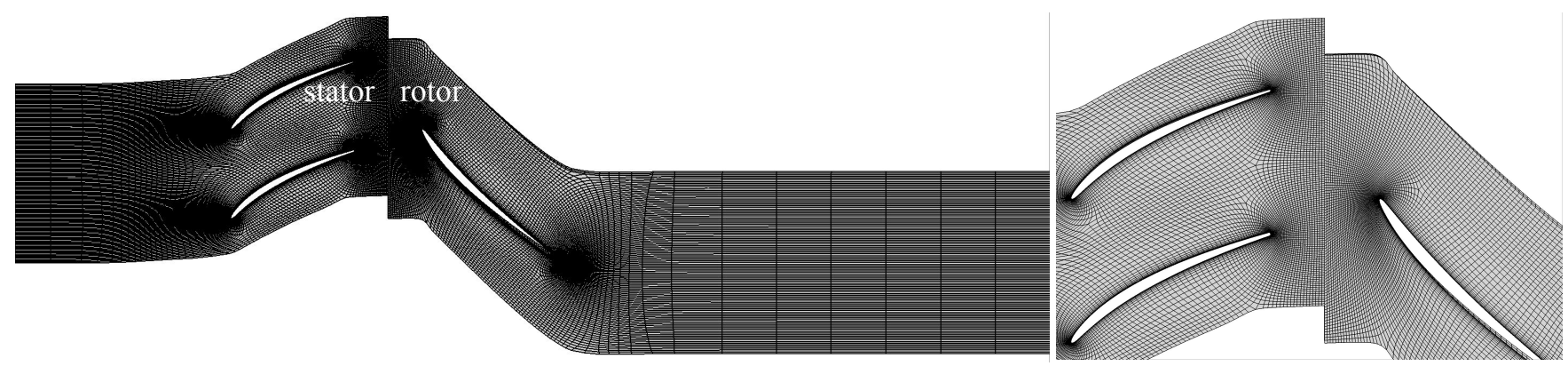

Fig. 4 Geometry and mesh of stator-rotor configuration.
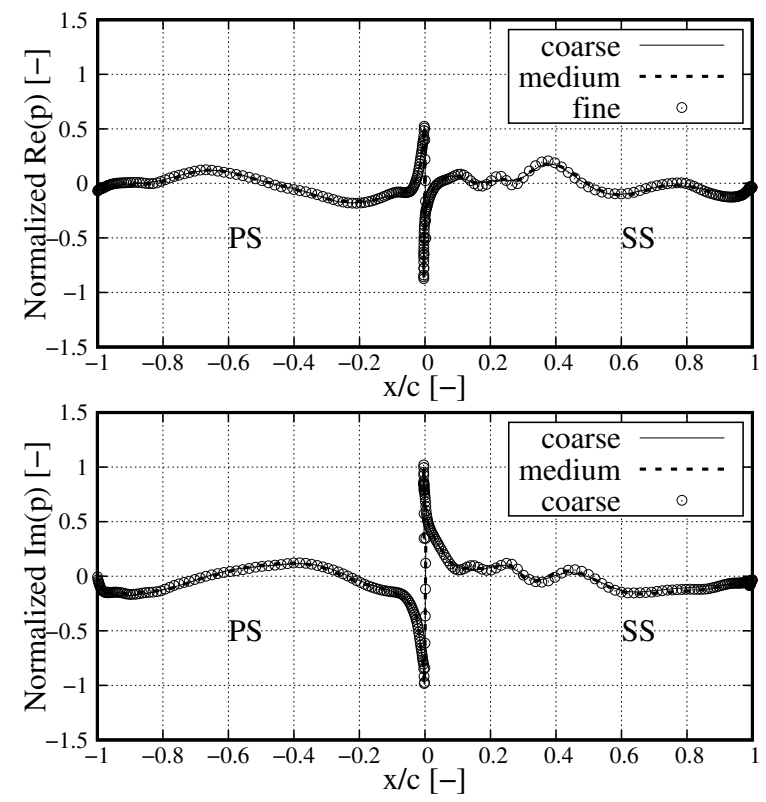

Fig. 5 Grid convergence study of the rotor-stator case. 


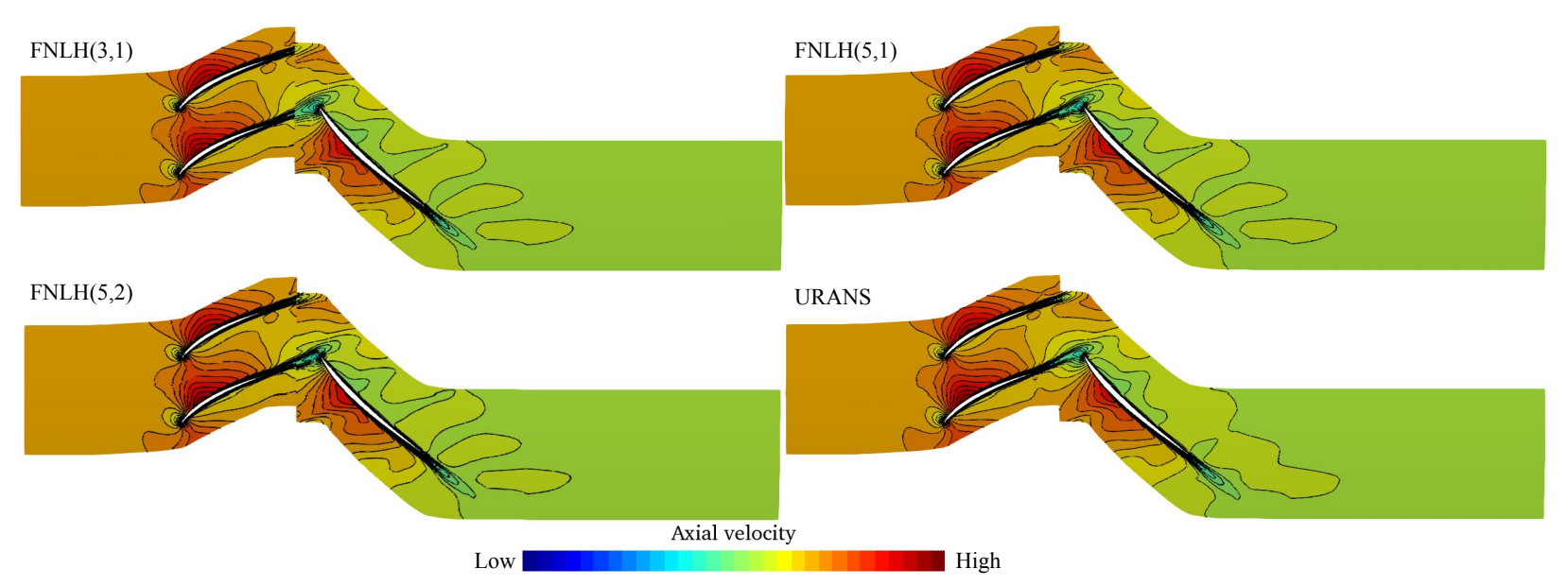

Fig. 6 Reconstructed unsteady velocity field for Quasi-3D cascades.

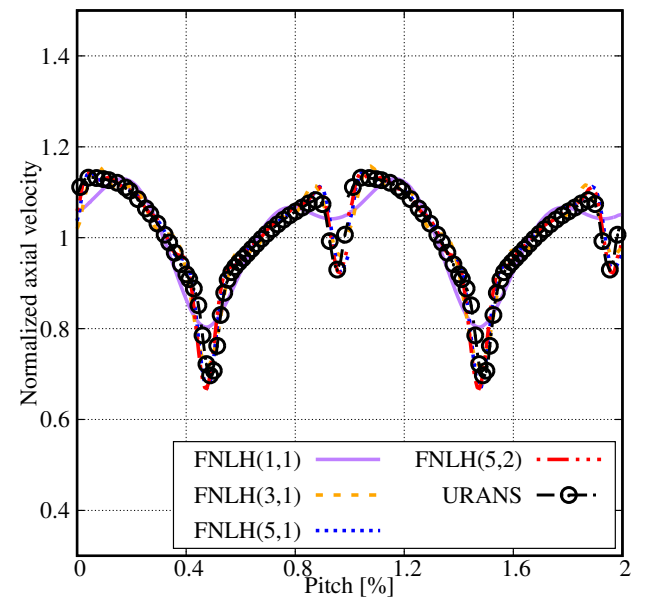

Fig. 7 Circumferential variation of axial velocity in front of rotor leading edge .

The reconstructed unsteady field of the axial velocity is shown in Fig. 6 and the circumferential variation of the axial velocity in front of the rotor leading edge is also presented in Fig. 7 The axial position where the axial velocity is extracted lies in the midway between the rotor inlet and leading edge. The two values in the bracket represent the number of harmonics to solve at the inlet and exit respectively. The first value in the bracket is the number of harmonics for the wake from upstream bladerow if present and the second is the number of harmonics for the potential effect from downstream blade row if present. It can be seen from Fig. 6 that the combination of $(3,1)$ provides a credible reconstruction of the unsteady axial velocity. This can also be seen from the circumferential variable of the axial velocity shown in Fig. 77 The general shape of the wake is well resolved by the combination of $(3,1)$ and adding more harmonics only provides a more detailed representation of the depth of the wake. Besides, the combination of $(5,2)$ improves slightly the flow continuity of the unsteady flow on the bladerow interface but has little effect on the wake in the rotor passage as is shown in Fig. 7

Due to the upstream stator wake, the pressure distribution on the rotor blade surface changes periodically. Because 

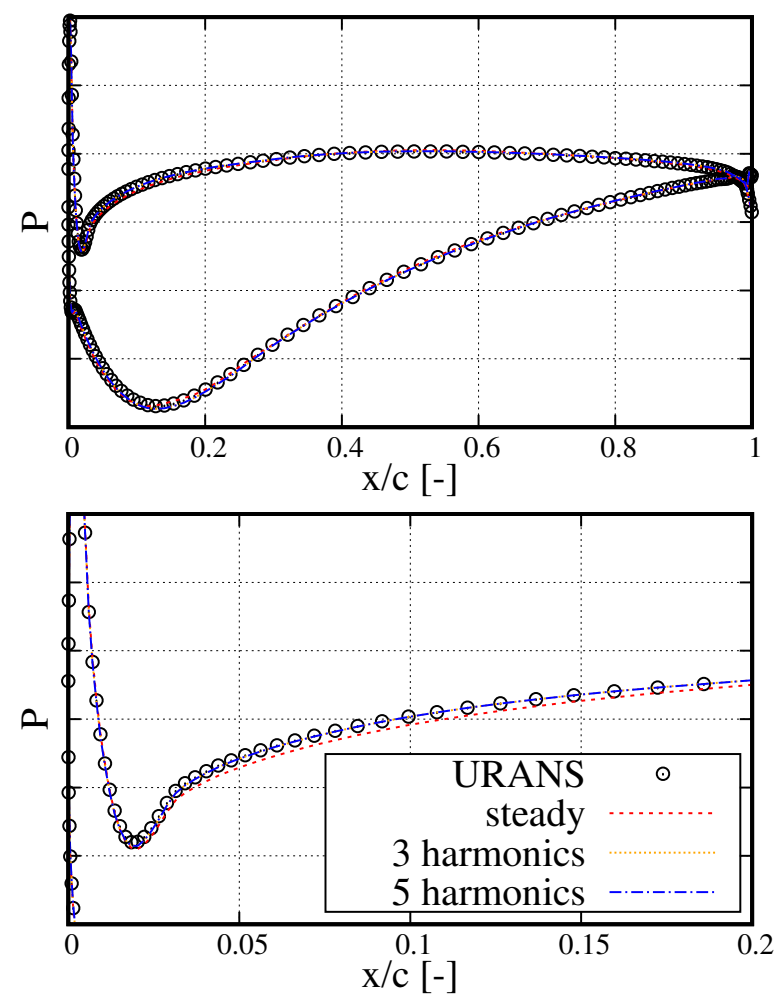

Fig. 8 Time-averaged pressure on the rotor blade surface.

the NS equations are non-linear, the pressure distribution of the time mean flow on the blade surface will be different from that of the steady mixing plane approach. Figure 8 shows the static pressure distribution on the blade. The pressure distribution from the current method matches well with the distribution from the time-averaged solution. Similar to the observation in the unsteady axial velocity field, 3 harmonics are sufficient to obtain credible agreement with the URANS.

The unsteady pressure on the blade is also compared with the URANS data. Figure 9 shows the real and imaginary part of the first harmonic of the unsteady pressure on the rotor blade surface. Because the cross-coupling among the harmonics is not considered in this work, including higher harmonics has marginal effect on the solution of the first harmonic. The predicted unsteady pressure has a good agreement with the URANS data.

\section{3D Multistage transonic Compressor}

The third case is the front 1.5 stages of a multistage compressor which was previously studied by the authors [30]. The flow in the rotor is in the transonic flow regime. It is most interesting to see the difference of the predicted compressor performance (e.g. efficiency and pressure ratio) using the steady mixing plane approach and FNLH which includes deterministic fluxes. This is instructive for the engine designers to appreciate the significance of unsteady effects on the prediction of compressor performance [32]. 

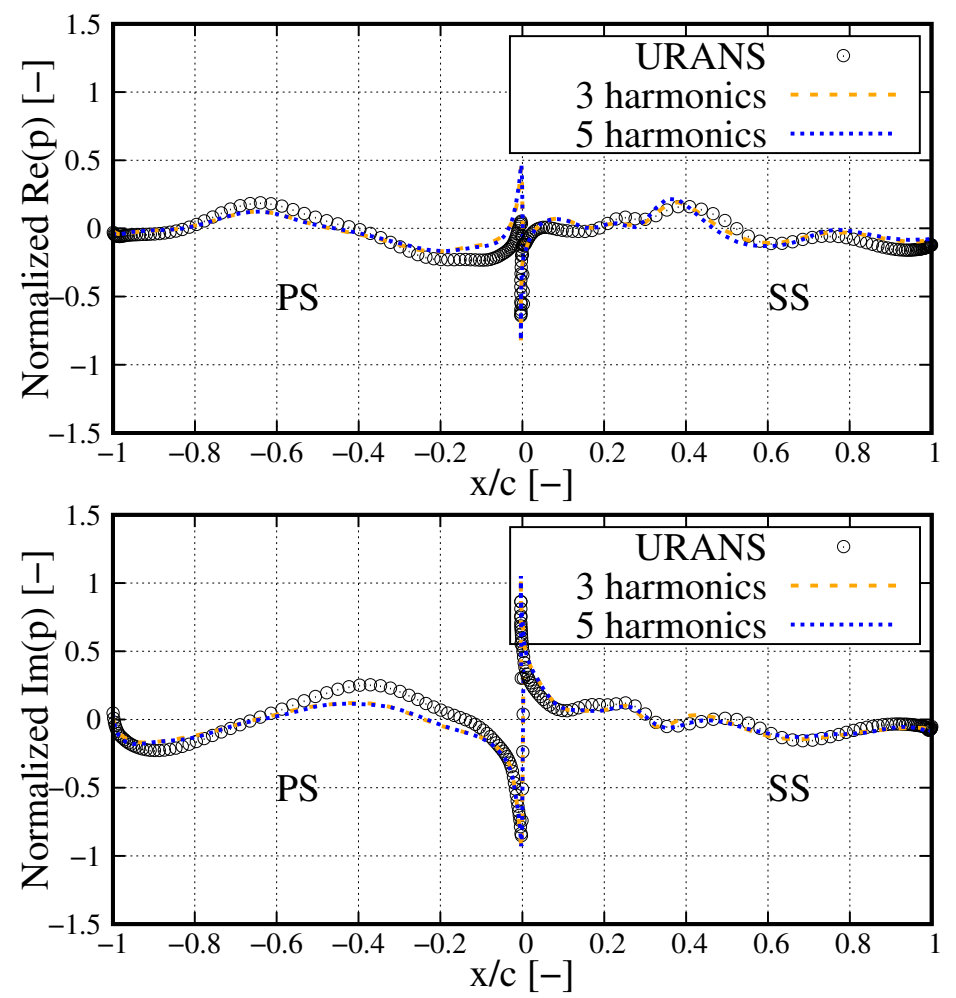

Fig. 9 First harmonic of unsteady pressure on the rotor blade surface.

The geometry is shown in Fig. 10 a) and the blade counts have been modified so that a $15^{\circ}$ sector can be simulated for the URANS. Due to the modification of the geometry, the predicted performance and flow fields do not necessarily represent the ones of the original machine. The inlet boundary of the upstream stator and the exit of the downstream stator have been extended and the meshes around those regions have been coarsened in the streamwise direction to damp spurious reflective waves in the URANS. The boundary conditions for the inlet and exit boundaries are taken from a full compressor simulation. The back pressure is varied to obtain the compressor map under different mass flow rates.

The size of the mesh is based on the grid convergence study of the previous work by Wang et al. [30]. Each passage has around 0.9 million cells and the tip gap of the rotor is discretized with 15 layers. The $y^{+}$close to the viscous wall is around 1. Automatic wall treatment is used so that the solution is relatively insensitive to the near wall grid spacing. The mesh for the URANS is generated by replicating the meshes for the steady mixing plane in the circumferential direction. 150 time steps are used for the rotor to pass the stator. This is 450 time steps for the $15^{0}$ sector and is equivalent to 10,800 time steps if the whole wheel is the case. The choice of the time step is based on the previous URANS study of the same compressor [33]. The mesh for the FNLH is identical to the steady simulation with mixing plane.

Figure 11 shows the comparison of the predicted performance maps for the current method, URANS with sliding plane and steady simulation with mixing plane. The total quantities of the URANS data are computed from the Favre-averaged primitive variables. MRTP is the corrected mass flow and is computed based on the total quantities at 

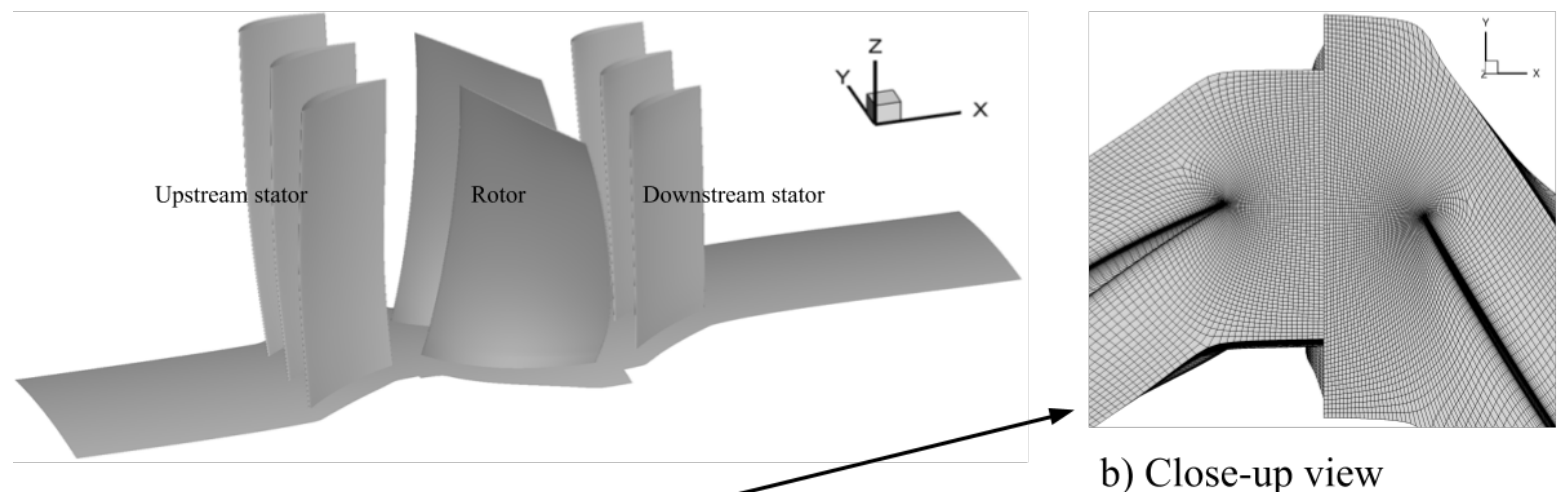

a) Geometry

b) Close-up view

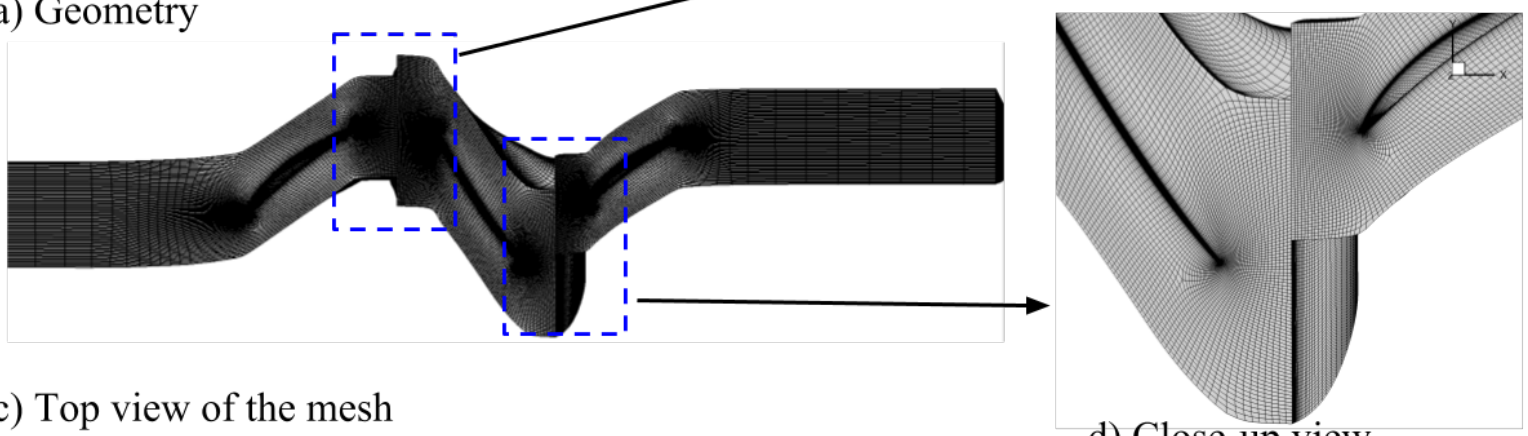

d) Close-up view

Fig. 10 Mesh details of the multistage compressor configuration.

the inlet of each bladerow. To understand the unsteady effect on each bladerow, the performance data of the rotor and the downstream stator are shown separately. Spectral convergence of FNLH with different harmonics is also shown in the figure, which is informative for designers to deploy the current method in an cost-effective manner.

An excellent agreement between FNLH and the URANS is observed for both the pressure ratio and efficiency. Besides, it is interesting to see in Fig. 11 that:

- The pressure ratio of the stator is smaller than 1 since there is no work input. The total pressure ratio is an indirect measure of the loss. It is interesting to see that with the unsteady effects included in the mean flow fewer losses are observed. This effect is mainly due to the wake recovery effect reported by Van Zante et al. [34].

- The total pressure ratio and efficiency of the rotor is also increased due to the presence of the upstream stator. This is due to the combined effect of the wake recovery and the interaction of the stator wake with the rotor tip leakage flow.

- The pressure ratio of the rotor is relatively insensitive to the increment of harmonics in the simulation. 3 harmonics are sufficient to obtain a credible agreement with the unsteady simulations. However the efficiency shows a higher sensitivity on the number of harmonics. This is consistent with the results reported by Gudeney et al. [35] using the harmonic balance technique to simulate a 1.5 stage compressor. For the downstream stator, the pressure ratio is also sensitive to the number of harmonics and share the same trend of the rotor efficiency. The current results 


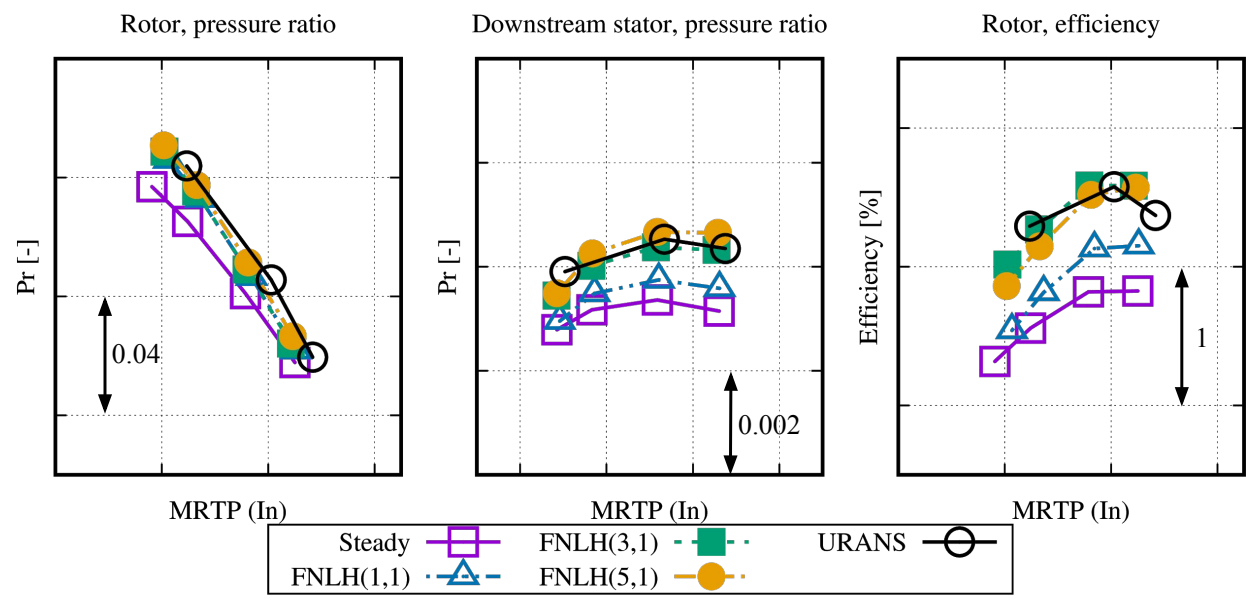

Fig. 11 Spectral convergence and predicted compressor performance by FNLH.

strongly indicate that more harmonics are required for a better reconstruction of rotor efficiency and the loss in the stator.

Figure 12 shows the reconstructed unsteady field for pressure and entropy using FNLH. The pressure field due to the shock on the rotor is reconstructed satisfactorily compared to the unsteady simulation, despite the fact that only one harmonic is used for the potential effect from the rotor to the upstream stator. This is because the shock in the rotor is diffused when propagating upstream and higher harmonics are damped. For compressors with more compact axial spacing, more harmonics are required to resolve these potential effects. Regarding to entropy distribution, clocking effect is not considered in this case, so the wake of the upstream stator is not computed in the downstream stator. Nevertheless, this is found to have minor effect on the performance of the downstream stator.

\section{Conclusions}

This paper has studied the averaging procedure on periodic compressible flows with finite magnitude. The choice of variable set implies a specific choice of average: as an example, working with primitive variables leads to the evaluation of Favre-averages, whereas working with conserved variables leads to the evaluation of time averages of those variables. Consistency of the mean flow definition is preserved by the mutual relations between Favre and time averages. As an example, for the velocities

$$
\bar{\rho} \tilde{u}_{i}=\overline{\rho u_{i}}
$$

The choice of averaging, however, leads to two non-equivalent sets of perturbation variables which need to be treated consistently when evaluating linearized fluxes and residuals.

Favre-averaging provides a simpler formulation of the time-averaged NS equations and a new formulation of the non-linear harmonic method is proposed based on Favre-averaging. The resulting formulation can lead to minimal 

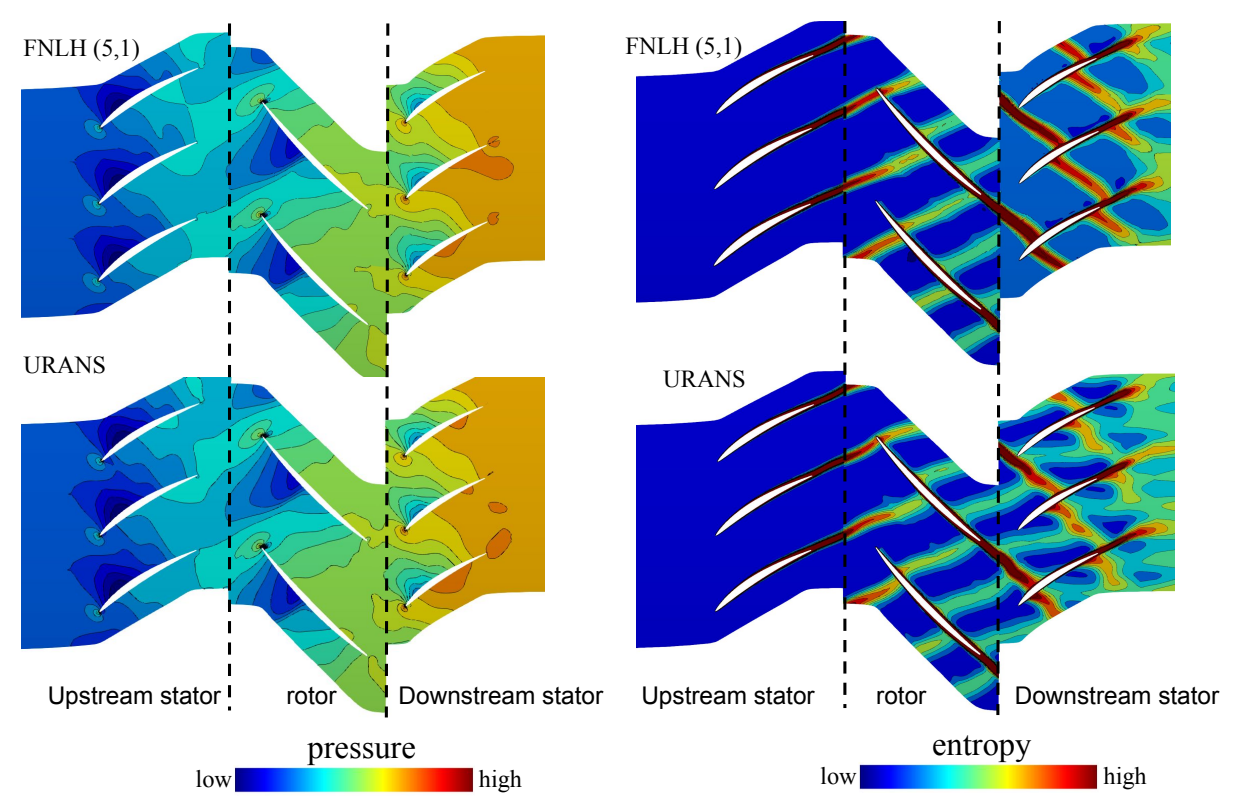

Fig. 12 Reconstructed unsteady flow field around middle span.

modifications to the baseline solver to implement the non-linear harmonic method.

The proposed method has been described in detail and also been validated on a transonic diffuser, on quasi-3D rotor-stator simulation and on 3D multistage compressors. Good agreement with the URANS solutions are observed. In the compressor simulations, it is found that typically three harmonics are sufficient to gain good agreement with the URANS data. This is because the deterministic fluxes scale quadratically with the magnitude of the flow perturbations and the magnitude of the harmonics decreases when the harmonic index is increased. The benifit of including higher harmonics on the mean flow decays quickly as more harmonics are considered.

\section{Acknowledgement}

The authors would like to thank Rolls-Royce plc for funding this work through the GEMinIDS project and permitting its publication.

\section{References}

[1] Adamczyk, J. J., "Model equation for simulating flows in multistage turbomachinery," Tech. Rep. NASA-TM-86869, NASA Lewis Research Center, Cleveland, OH, United States, Nov 1984.

[2] Adamczyk, J. J., “Aerodynamic Analysis of Multistage Turbomachinery Flows in Support of Aerodynamic Design,” Journal of Turbomachinery, Vol. 122, No. 2, 2000, p. 189. doi:10.1115/1.555439, URL https://doi .org/10.1115/1.555439.

[3] ???? 
[4] He, L., and Ning, W., "Efficient Approach for Analysis of Unsteady Viscous Flows in Turbomachines," AIAA Journal, Vol. 36, No. 11, 1998, pp. 2005-2012. doi:10.2514/2.328, URL https://doi.org/10.2514/2.328

[5] Vasanthakumar, P., "Three dimensional frequency-domain solution method for unsteady turbomachinery flows," Phd thesis, Durham University, Durham, UK, Jan 2003.

[6] He, L., Chen, T., Wells, R. G., Li, Y. S., and Ning, W., “Analysis of Rotor-Rotor and Stator-Stator Interferences in Multi-Stage Turbomachines," Journal of Turbomachinery, Vol. 124, No. 4, 2002, p. 564. doi:10.1115/1.1508382, URL https://doi.org/10.1115/1.1508382

[7] Hall, K. C., Thomas, J. P., and Clark, W. S., "Computation of Unsteady Nonlinear Flows in Cascades Using a Harmonic Balance Technique," AIAA Journal, Vol. 40, No. 5, 2002, pp. 879-886. doi:10.2514/2.1754, URL https://doi.org/10.2514/2. 1754

[8] Sicot, F., Puigt, G., and Montagnac, M., "Block-Jacobi Implicit Algorithms for the Time Spectral Method," AIAA Journal, Vol. 46, No. 12, 2008, pp. 3080-3089. doi:10.2514/1.36792, URL https://doi.org/10.2514/1.36792

[9] McMullen, M., and Jameson, A., “The computational efficiency of non-linear frequency domain methods," Journal of Computational Physics, Vol. 212, No. 2, 2006, pp. 637-661. doi:10.1016/j.jcp.2005.07.021, URL https://doi.org/10. $1016 / j \cdot j c p .2005 .07 .021$

[10] Hall, K. C., Ekici, K., Thomas, J. P., and Dowell, E. H., "Harmonic balance methods applied to computational fluid dynamics problems," International Journal of Computational Fluid Dynamics, Vol. 27, No. 2, 2013, pp. 52-67. doi: 10.1080/10618562.2012.742512, URL https://doi.org/10.1080/10618562.2012.742512

[11] Thomas, J. P., Custer, C. H., Dowell, E. H., Hall, K. C., and Corre, C., "Compact Implementation Strategy for a Harmonic Balance Method Within Implicit Flow Solvers,” AIAA Journal, Vol. 51, No. 6, 2013, pp. 1374-1381. doi:10.2514/1.j051823, URL https://doi .org/10.2514/1.j051823

[12] Vilmin, S., Lorrain, E., Debrabandere, F., Tartinville, B., Capron, A., and Hirsch, C., "The Nonlinear Harmonic Method Applied to the Combined Effects of Multi-Row Unsteady Flows," Volume 6C: Turbomachinery, ASME, 2013. doi:10.1115/gt2013-94847, URL https://doi.org/10.1115/gt2013-94847

[13] Chen, T., Vasanthakumar, P., and He, L., “Analysis of Unsteady Blade Row Interaction Using Nonlinear Harmonic Approach,” Journal of Propulsion and Power, Vol. 17, No. 3, 2001, pp. 651-658. doi:10.2514/2.5792, URL https://doi.org/10. $2514 / 2.5792$

[14] Vilmin, S., Lorrain, E., Hirsch, C., and Swoboda, M., "Unsteady Flow Modeling Across the Rotor/Stator Interface Using the Nonlinear Harmonic Method," Volume 6: Turbomachinery, Parts A and B, ASME, 2006. doi:10.1115/gt2006-90210, URL https://doi.org/10.1115/gt2006-90210 
[15] Vilmin, S., Lorrain, É., Tartinville, B., Capron, A., and Hirsch, C., "The nonlinear harmonic method: from single stage to multi-row effects," International Journal of Computational Fluid Dynamics, Vol. 27, No. 2, 2013, pp. 88-99. doi: 10.1080/10618562.2012.752074, URL https://doi.org/10.1080/10618562.2012.752074

[16] Wilcox, D. C., Turbulence Modeling for CFD (Third Edition), D C W Industries, 2006. URL https://www . amazon. com/ Turbulence-Modeling-Third-David-Wilcox/dp/1928729088?SubscriptionId=0JYN1NVW651KCA56C102\&tag= techkie-20\&linkCode $=x m 2 \&$ camp $=2025 \&$ creative $=165953 \&$ creativeASIN $=1928729088$

[17] Cebeci, T., Turbulence Models and Their Application: Efficient Numerical Methods with Computer Programs, Springer, 2004. URL https://wWw.amazon.com/Turbulence-Models-Their-Application-Efficient/dp/3540402888? SubscriptionId=0JYN1NVW651KCA56C102\&tag=techkie-20\&linkCode=xm2\&camp=2025\&creative=165953\& creativeASIN $=3540402888$

[18] di Mare, L., Kulkarni, D. Y., Wang, F., Romanov, A., Ramar, P. R., and Zachariadis, Z. I., "Virtual Gas Turbines: Geometry and Conceptual Description," Proceedings of ASME TurboExpo, Vancouver, Canada, ASME, 2011. doi:10.1115/gt2011-46437, URL https://doi .org/10.1115/gt2011-46437

[19] Wang, F., Carnevale, M., Lu, G., di Mare, L., and Kulkarni, D., "Virtual Gas Turbine: Pre-Processing and Numerical Simulations," Proceedings of ASME TurboExpo, South Korea, Seoul, ASME, 2016. doi:10.1115/gt2016-56227, URL https://doi.org/10.1115/gt2016-56227

[20] Carnevale, M., Wang, F., Green, J. S., and Mare, L. D., "Lip Stall Suppression in Powered Intakes,” Journal of Propulsion and Power, Vol. 32, No. 1, 2016, pp. 161-170. doi:10.2514/1.b35811, URL https://doi.org/10.2514/1.b35811.

[21] Carnevale, M., Wang, F., Parry, A. B., Green, J. S., and di Mare, L., "Fan Similarity Model for the Fan-Intake Interaction Problem," Journal of Engineering for Gas Turbines and Power, Vol. 140, No. 5, 2017, p. 051202. doi:10.1115/1.4038247, URL https://doi.org/10.1115/1.4038247

[22] Hadade, I., Wang, F., Carnevale, M., and di Mare, L., "Some useful optimisations for unstructured computational fluid dynamics codes on multicore and manycore architectures," Computer Physics Communications, 2018. doi:10.1016/j.cpc.2018.07.001, URL https://doi.org/10.1016/j.cpc.2018.07.001

[23] Mavriplis, D., "Revisiting the Least-Squares Procedure for Gradient Reconstruction on Unstructured Meshes," 16th AIAA Computational Fluid Dynamics Conference, American Institute of Aeronautics and Astronautics, 2003. doi:10.2514/6.2003-3986, URL https://doi.org/10.2514/6.2003-3986.

[24] Blazek, J., Computational fluid dynamics : principles and applications, Butterworth Heinemann, Amsterdam San Diego, 2015.

[25] Giles, M., "Non-Reflecting Boundary Conditions for the Euler Equations," Tech. Rep. CFDL-TR-88-1, Massachusetts Institute of Technology, feb 1988. 
[26] Saxer, A. P., and Giles, M. B., "Quasi-three-dimensional nonreflecting boundary conditions for Euler equations calculations," Journal of Propulsion and Power, Vol. 9, No. 2, 1993, pp. 263-271. doi:10.2514/3.23618, URL https://doi.org/10. $2514 / 3.23618$

[27] Denton, J. D., “The Calculation of Three-Dimensional Viscous Flow Through Multistage Turbomachines," Journal of Turbomachinery, Vol. 114, No. 1, 1992, p. 18. doi:10.1115/1.2927983, URL https://doi.org/10.1115/1.2927983

[28] F. R. Menter, M. K., and Langtry, R., “Ten Years of Industrial Experience with the SST Turbulence Model,” Proceedings of the 4th International Symposium on Turbulence, Heat and Mass Transfer, Begell House Inc., West Redding, USA, 2003, pp. 625-632.

[29] Sajben, M., Bogar, T., and Kroutil, J., "Forced oscillation experiments in supercritical diffuser flows with application to ramjet instabilities," 17th Joint Propulsion Conference, American Institute of Aeronautics and Astronautics, 1981. doi: 10.2514/6.1981-1487, URL https://doi.org/10.2514/6.1981-1487

[30] Wang, F., Carnevale, M., di Mare, L., and Gallimore, S., "Simulation of Multistage Compressor at Off-Design Conditions," Journal of Turbomachinery, Vol. 140, No. 2, 2017, p. 021011. doi:10.1115/1.4038317, URL https://doi.org/10.1115/1. 4038317

[31] Wang, F., and di Mare, L., "Mesh Generation for Turbomachinery Blade Passages with Three-Dimensional Endwall Features," Journal of Propulsion and Power, Vol. 33, No. 6, 2017, pp. 1459-1472. doi:10.2514/1.b36356, URL https: //doi.org/10.2514/1.b36356

[32] Trebinjac, I., Kulisa, P., Bulot, N., and Rochuon, N., "Effect of Unsteadiness on the Performance of a Transonic Centrifugal Compressor Stage," Journal of Turbomachinery, Vol. 131, No. 4, 2009, p. 041011. doi:10.1115/1.3070575, URL https: //doi.org/10.1115/1.3070575

[33] Wang, F., Carnevale, M., and di Mare, L., "Numerical study of deterministic fluxes in compressor passages," Proceedings of ASME Turbo Expo 2017: Turbomachinery Technical Conference and Exposition, 2018, pp. 1-11. GT2018-76668.

[34] Zante, D. E. V., Adamczyk, J. J., Strazisar, A. J., and Okiishi, T. H., "Wake Recovery Performance Benefit in a HighSpeed Axial Compressor," Journal of Turbomachinery, Vol. 124, No. 2, 2002, p. 275. doi:10.1115/1.1445793, URL https://doi.org/10.1115/1.1445793

[35] Guédeney, T., Gomar, A., Gallard, F., Sicot, F., Dufour, G., and Puigt, G., "Non-uniform time sampling for multiple-frequency harmonic balance computations," Journal of Computational Physics, Vol. 236, 2013, pp. 317-345. doi:10.1016/j.jcp.2012.11.010, URL https://doi.org/10.1016/j.jcp.2012.11.010 